DOI https://doi.org/10.24297/jam.v19i.8834

\title{
Automorphisms of Zero Divisor Graphs of Square Radical Zero Commutative Unital Finite Rings
}

\author{
Lao Hussein Mude ${ }^{1}$, Owino Maurice Oduor ${ }^{2}$, Ojiema Michael Onyango ${ }^{3}$ \\ 1,2 Department of Mathematics and Computer Science, University of Kabianga, P. O. Box \\ 2030-20200, Kericho, Kenya . \\ ${ }^{3}$ Department of Mathematics, Masinde Muliro University of Science and Technology, P. O. Box \\ 190-50100, Kakamega, Kenya. \\ ${ }^{1}$ hlao@kabianga.ac.ke, ${ }^{2}$ modour@kabianga.ac.ke, ${ }^{3}$ mojiema@mmust.ac.ke
}

\begin{abstract}
There has been extensive research on the structure of zero divisors and units of commutative finite rings. However, the classification of such rings via a well known structure of zero divisors has not been done in general. More specifically, the automorphisms of such classes of rings have not been fully characterized. In this paper we obtain a more complete illustration of the automorphisms of zero divisor graphs of finite rings in which the product of any zero divisor is zero.
\end{abstract}

Keywords: Automorphisms, Zero Divisor Graphs, Square Radical Zero Finite Rings. .

Introduction The classification of completely primary finite rings has received much attention in the recent years. For related studies see $[1,2,3,4,5,6]$. Most scholars have concentrated in obtaining the structures of zero divisor graphs of commutative finite rings. However, little research has been done on the automorphisms of zero divisor graphs of completely primary finite rings, despite the fact that automorphisms have played practical role in understanding complexity of many algebraic structures. In view of the above, this research investigated the automorphsms of zero divisor graphs of square radical zero commutative unital finite rings. In this study, $R$ shall denote commutative finite rings as constructed in the sequel, $Z(R)$ will represent the Jacobson radical of $R$, the automorphism of the zero divisor graph of the ring $R$ will be denoted by $\operatorname{Aut}(\Gamma(R))$. The edge between vertices of a graph will be denoted $E$ and $|E|$ will represent the cardinality of the graph while $v$ denote a vertex in a graph. The cardinality of the automorphism group shall be denoted by $|A u t(\Gamma(R))|$. Unless otherwise given, $G R\left(p^{n r}, p^{n}\right)$ will denote Galois ring of order $p^{n r}$ and characteristic $p^{n}$ and it is uniquely determined by the invariant $p, n$ and $r$. The following result due to Raghavendran will be useful in the sequel.

\section{Results and Discussion}

\section{Preliminary Results}

Theorem 1.1. ([5] pp 99) Let $R$ be a finite ring with multiplicative identity $1 \neq 0$ whose zero divisors form an additive group $Z(R)$. Then,

(i) $Z(R)$ is the Jacobson radical of $R$

(ii) $|R|=p^{n r}$, and $|Z(R)|=p^{(n-1) r}$ for some prime integer $p$ and some positive integers $n$ and $r$ 
(iii) $(Z(R))^{n}=(0)$

(iv) The characteristic of a ring $R$ if $p^{k}$ for some positive integer $k$ with $1 \leq k \leq n$; and

(v) If the characteristic is $p^{n}$, then $R$ is commutative.

We begin with construction of the ring which can be obtained in [5].

\section{The Construction of Square Radical Zero Finite Rings}

For every prime integer $p$ and positive integer $r$, let $R_{0}=G F\left(p^{k r}, p^{k}\right)$ where $k=1,2$. For all $i=1, \ldots, h$, let $u_{i} \in Z(R)$ and $U$ is an $h$-dimensional $R_{0}$-module generated by $\left\{u_{1}, \ldots, u_{h}\right\}$. Suppose $R=R_{0} \oplus U$ is an additive group, it can easily be shown that the multiplication

$\left(a_{0}, a_{1}, \ldots, a_{h}\right)\left(b_{0}, b_{1}, \ldots, b_{h}\right)=\left(a_{0} b_{0}, a_{0} b_{1}+a_{1} b_{0}, \ldots, a_{0} b_{h}+a_{h} b_{0}\right)$, turns $R$ into a ring with identity $(1,0,0, \ldots 0)$. Since the results are different for each characteristics of the ring $R$, we present them on case to case basis.

\section{Characteristic of $R$ is $p$}

Proposition 1. Let $R$ be a ring of characteristic $p$ in the Construction. Then,

$$
|A u t(\Gamma(R))|=\frac{1}{p^{h r-1}} \Phi\left(p^{h r}\right)\left(p^{h r}-2\right) ! \sum_{i=1}^{h r} p^{h r-i} .
$$

Proof. In the above construction we consider $k=1$ so that $R_{0}=G R\left(p^{r}, p\right)$. Let $K=R_{0} / p R_{0}$ be the field. Suppose $U=K^{h}$ is an $R_{0}$-module generated by $\left\{u_{1}, u_{2}, u_{3}, \cdots, u_{h}\right\}$. Therefore $R=R_{0} \oplus U$ is an additive abelian group. But $Z(R)=R_{0} u_{1} \oplus R_{0} u_{2} \oplus \cdots \oplus R_{0} u_{h}$ and every element in $Z(R)^{*}$ is of the form $\left(0, a_{1}, a_{2}, \cdots, a_{h}\right)$ so that the product of every pair $\left(0, a_{1}, a_{2}, \cdots, a_{h}\right)$,

$\left(0, b_{1}, b_{2}, \cdots, b_{h}\right) \in Z(R)^{*}$ is identically zero, indicating that every pair of elements of zero divisor graph of $R$ are joined by an edge. So $|V(\Gamma(R))|=\left|Z(R)^{*}\right|=p^{h r}-1$ so that $\operatorname{Aut}(\Gamma(R)) \cong S_{p^{h r}-1}$. Since $|A u t(\Gamma(R))|=$ $\left(p^{h r}-1\right) !=\left(p^{h r}-1\right)\left(p^{h r}-2\right) !=(p-1) \sum_{i=1}^{h r} p^{h r-i}\left(p^{h r}-2\right) !$ and $\Phi\left(p^{h r}\right)=p^{h r}-p^{h r-1}=p^{h r-1}(p-1)$. Dividing $|A u t(\Gamma(R))|$ by $\Phi\left(p^{h r}\right)$ and expressing the relation in terms of $|A u t(\Gamma(R))|$ establishes the relation $|A u t(\Gamma(R))|=$ $\frac{1}{p^{h r-1}} \Phi\left(p^{h r}\right)\left(p^{h r}-2\right) ! \sum_{i=1}^{h r} p^{h r-i}$.

Corollary 3.1. Let $R$ be a ring in the construction and of characteristic $p$. Then,

$$
|A u t(\Gamma(R))|=|V(\Gamma(R))|\left(p^{h r}-2\right) !
$$

Proof. This is a consequence of Proposition 1.

Proposition 2. Let $R$ be a ring of characteristic $p$ in the construction. Then,

$$
|V(\Gamma(R))|=\frac{1}{p^{h r-1}} \Phi\left(p^{h r}\right) \sum_{i=1}^{h r} p^{h r-i} .
$$


Proof. We note that $|V(\Gamma(R))|=p^{h r}-1=(p-1)\left(p^{h r-1}+p^{h r-2}+p^{h r-3}+\cdots+1\right)=(p-1) \sum_{i=1}^{h r} p^{h r-i}$. But $\Phi\left(p^{h r}\right)=$ $p^{h r}-p^{h r-1}=p^{h r-1}(p-1)$. Making $|V(\Gamma(R))|$ the subject of the formula gives $|V(\Gamma(R))|=\frac{1}{p^{h r-1}} \Phi\left(p^{h r}\right) \sum_{i=1}^{h r} p^{h r-i}$.

Proposition 3. Let $R$ be a ring of characteristic $p$, in the Construction. Then,

$$
|E|=\frac{1}{2}\left(p^{h r}-1\right)\left(p^{h r}-2\right) .
$$

Proof. By Proposition 1, $\Gamma(R)$ is complete on $\left(p^{h r}-1\right)$ vertices. For each $\left(p^{h r}-1\right)$ vertices of $\Gamma(R)$, label the vertices $1,2,3, \cdots,\left(p^{h r}-1\right)$. The first vertex will be adjacent to $\left(p^{h r}-2\right)$ other vertices. The second one will be connected to the remaining $\left(p^{h r}-3\right)$ vertices and more generally the $n^{\text {th }}$ vertex will be connected to the remaining $\left(p^{h r}-n\right)$ vertices. Therefore, the sum of the edges in $\Gamma(R)$ is given by $\left.\left.\left(p^{h r}-2\right)+\left(p^{h r}-3\right)+\left(p^{h r}-4\right)+\cdots+2+1\right)\right)=\frac{1}{2}\left(p^{h r}-1\right)\left(p^{h r}-2\right)$ completing the proof.

Proposition 4. Let $R$ be a ring of characteristic $p$ in the Construction. Then,

$$
\sum_{v \in V} \operatorname{deg}(v)=\left(p^{h r}-1\right)\left(p^{h r}-2\right)
$$

Proof. Evidently, from Proposition 1 every vertex in $\Gamma(R)$ is connected to other. Each edge is incident to two other vertices in $\Gamma(R)$. Thus, the number of degrees will double. So, for $E$ such edges, the sum of the degrees is twice the number of edges.

Proposition 5. Let $R$ be a ring of characteristic $p$ in the Construction. Then,

$$
|A u t(\Gamma(R))|=\left\{\begin{array}{l}
\left(p^{h r}-3\right) ! \sum_{v \in V} \operatorname{deg}(v) . \\
2\left(p^{h r}-3\right) !|E| .
\end{array}\right.
$$

Proof. By Proposition $1|A u t(\Gamma(R))|=\left(p^{h r}-1\right)$ ! and the sum of degrees of zero divisor graph of $R$ is $\left(p^{h r}-1\right)\left(p^{h r}-2\right)$ as stated in the above result. Since $\frac{\left(p^{h r}-1\right) !}{\left(p^{h r}-1\right)\left(p^{h r}-2\right)}=\left(p^{h r}-3\right)$ !. it follows that $|A u t(\Gamma(R))|=\left(p^{h r}-3\right) ! \sum_{v \in V} d e g(v)$. By Proposition $1|A u t(\Gamma(R))|=\left(p^{h r}-1\right)$ ! and from the above Proposition, the sum of edges of $\Gamma(R)$ is $\frac{1}{2}\left(p^{h r}-1\right)\left(p^{h r}-2\right)$. Since $\frac{\left(p^{h r}-1\right) !}{\frac{1}{2}\left(p^{h r}-1\right)\left(p^{h r}-2\right)}=2\left(p^{h r}-3\right)$ ! we obtain $|A u t(\Gamma(R))|=2\left(p^{h r}-3\right) !|E|$.

Proposition 6. Let $R$ be a ring of characteristic $p$ in the Construction. Then,

$$
|V(\Gamma(R))|=\left\{\begin{array}{l}
\frac{1}{p^{h r}-2} \sum_{v \in V} \operatorname{deg}(v) . \\
\frac{2|E|}{p^{h r}-2} .
\end{array}\right.
$$

Proof. $\left|Z(R)^{*}\right|=|V(\Gamma(R))|=p^{h r}-1$ and the sum of the degrees of $\Gamma(R)$ is $\left(p^{h r}-1\right)\left(p^{h r}-2\right)$. Since $\frac{\left(p^{h r}-1\right)}{\left(p^{h r}-1\right)\left(p^{h r}-2\right)}=$ $\frac{1}{\left(p^{h r}-2\right)}$ the result easily follows.

The sum of edges of $\Gamma(R)$ is $\frac{1}{2}\left(p^{h r}-1\right)\left(p^{h r}-2\right)$ follows from the above Proposition while $\left|Z(R)^{*}\right|=|V(\Gamma(R))|=p^{h r}-1$. Since $\frac{\left(p^{h r}-1\right)}{\frac{1}{2}\left(p^{h r}-1\right)\left(p^{h r}-2\right)}=\frac{2}{\left(p^{h r}-2\right)}$ the result is obvious. 


\section{Characteristics of $R$ is $p^{2}$}

Proposition 7. Let $R$ be a ring in the Construction of characteristic $p^{2}$. Then,

$$
|A u t(\Gamma(R))|=\frac{1}{p^{(h+1) r-1}} \Phi\left(p^{(h+1) r}\right)\left(p^{(h+1) r}-2\right) ! \sum_{i=1}^{(h+1) r} p^{(h+1) r-i} .
$$

Proof. In the above Construction, we have $R_{0}=G R\left(p^{2 r}, p^{2}\right)$ and $K=R_{0} / p R_{0}$. Let $U=K^{h}$ be an $R_{0}$-module generated by $\left\{u_{1}, u_{2}, u_{3}, \cdots, u_{h}\right\}$ and $R=R_{0} \oplus U$ is an additive group. Clearly, $Z(R)=p R_{0} \oplus R_{0} u_{1} \oplus R_{0} u_{2} \oplus \cdots \oplus R_{0} u_{h}$ and the product of every pair of elements in $Z(R)$ is zero. Thus $|R|=\left|R_{0}\right||U|=p^{(h+2) r} \Rightarrow|Z(R)|=p^{(h+1) r}$ and $|V(\Gamma(R))|=\left|Z(R)^{*}\right|=p^{(h+1) r}-1$. Since every element of $Z(R)^{*}$ is adjacent to all the other vertices of the zero divisor graph of $R$, so $\operatorname{Aut}(\Gamma(R))$ must permute all the symmetries of $\Gamma(R)$ independently so that $\operatorname{Aut}(\Gamma(R)) \cong$ $S_{p^{(h+1) r}-1}$. Since $|\operatorname{Aut}(\Gamma(R))|=\left(p^{(h+1) r}-1\right) !=\left(p^{(h+1) r}-1\right)\left(p^{(h+1) r}-2\right) !=(p-1) \sum_{i=1}^{(h+1) r} p^{(h+1) r-i}\left(p^{(h+1) r}-2\right) !$ and $\Phi\left(p^{(h+1) r}\right)=p^{(h+1) r}-p^{(h+1) r-1}=p^{(h+1) r-1}(p-1)$. Giving the relation in terms of $\mid A u t(\Gamma(R) \mid$ yields $|A u t(\Gamma(R))|=$ $\frac{1}{p^{(h+1) r-1}} \Phi\left(p^{(h+1) r}\right)\left(p^{(h+1) r}-2\right) ! \sum_{i=1}^{(h+1) r} p^{(h+1) r-i}$.

Corollary 4.1. Let $R$ be a ring in the Construction of characteristic $p^{2}$. Then, $|A u t(\Gamma(R))|=|V(\Gamma(R))|\left(p^{(h+1) r}-2\right)$ !.

Proof. The result follows from Proposition 7.

Proposition 8. Let $R$ be a ring of characteristic $p^{2}$ in the Construction. Then,

$$
|V(\Gamma(R))|=\frac{1}{p^{(h+1) r-1}} \Phi\left(p^{(h+1) r}\right) \sum_{i=1}^{(h+1) r} p^{(h+1) r-i} .
$$

Proof. $|V(\Gamma(R))|=p^{(h+1) r}-1=(p-1)\left(p^{(h+1) r-1}+p^{(h+1) r-2}+p^{(h+1) r-3}+\cdots+1\right)=(p-1) \sum_{i=1}^{(h+1) r} p^{(h+1) r-i}$ and $\Phi\left(p^{(h+1) r}\right)=p^{(h+1) r}-p^{(h+1) r-1}=p^{(h+1) r-1}(p-1)$, so that $|V(\Gamma(R))|=\frac{1}{p^{(h+1) r-1}} \Phi\left(p^{(h+1) r}\right) \sum_{i=1}^{(h+1) r} p^{(h+1) r-i}$.

Proposition 9. Let $R$ be a ring of characteristic $p^{2}$ in the Construction. Then, $|E|=\frac{1}{2}\left(p^{(h+1) r}-1\right)\left(p^{(h+1) r}-2\right)$. Moreover, $\sum_{v \in V} \operatorname{deg}(v)=\left(p^{(h+1) r}-1\right)\left(p^{(h+1) r}-2\right)$.

Proof. Follows from Proposition 3 and 5 with some slight changes.

Proposition 10. Let $R$ be a ring of characteristic $p^{2}$ in the Construction. Then,

$$
|\operatorname{Aut}(\Gamma(R))|=\left\{\begin{array}{l}
\left(p^{(h+1) r}-3\right) ! \sum_{v \in V} \operatorname{deg}(v) . \\
2\left(p^{(h+1) r}-3\right) !|E| .
\end{array}\right.
$$

Proof. By Proposition 7, $|\operatorname{Aut}(\Gamma(R))|=\left(p^{(h+1) r}-1\right)$ ! and $\left|Z(R)^{*}\right|=|V(\Gamma(R))|=\left(p^{(h+1) r}-1\right)$. But the sum of degrees $\Gamma(R)$ is $\left(p^{(h+1) r}-1\right)\left(p^{(h+1) r}-2\right)$. Since $\frac{\left(p^{(h+1) r}-1\right) !}{\left(p^{(h+1) r}-1\right)\left(p^{(h+1) r}-2\right)}=\left(p^{(h+1) r}-3\right) !$ it follows that $|A u t(\Gamma(R))|=$ $\left(p^{(h+1) r}-3\right) ! \sum_{v \in V} \operatorname{deg}(v)$.

By Proposition $7|\operatorname{Aut}(\Gamma(R))|=\left(p^{(h+1) r}-1\right)$ ! and $\left|Z(R)^{*}\right|=|V(\Gamma(R))|=\left(p^{(h+1) r}-1\right)$. But the sum of the edges of $\Gamma(R)$ is $\frac{1}{2}\left(p^{(h+1) r}-1\right)\left(p^{(h+1) r}-2\right)$. Since $\frac{\left(p^{(h+1) r}-1\right) !}{\frac{1}{2}\left(p^{(h+1) r}-1\right)\left(p^{(h+1) r}-2\right)}=2\left(p^{(h+1) r}-3\right) !$ we have $|A u t(\Gamma(R))|=2\left(p^{(h+1) r}-3\right) !|E|$. 
Proposition 11. Let $R$ be a ring of characteristic $p^{2}$ in the Construction. Then,

$$
|V(\Gamma(R))|=\left\{\begin{array}{l}
\frac{1}{p^{(h+) r}-2} \sum_{v \in V} \operatorname{deg}(v) . \\
\frac{2|E|}{p^{(h+1) r}-2} .
\end{array}\right.
$$

Proof. $\left|Z(R)^{*}\right|=\mid V\left(\Gamma(R) \mid=p^{(h+1) r}-1\right.$ and the sum of the degrees of $\Gamma(R)$ is $\left(p^{(h+1) r}-1\right)\left(p^{(h+1) r}-2\right)$. Since $\frac{\left(p^{(h+1) r}-1\right)}{\left(p^{(h+1) r}-1\right)\left(p^{(h+1) r}-2\right)}=\frac{1}{\left(p^{(h+1) r}-2\right)}$ the result is obvious.

$\left|Z(R)^{*}\right|=\mid V\left(\Gamma(R) \mid=p^{(h+1) r}-1\right.$ and the sum of edges of $\Gamma(R)$ is $\frac{1}{2}\left(p^{(h+1) r}-1\right)\left(p^{(h+1) r}-2\right)$. Since $\frac{\left(p^{(h+1) r}-1\right)}{\frac{1}{2}\left(p^{(h+1) r}-1\right)\left(p^{(h+1) r}-2\right)}=$ $\frac{2}{\left(p^{(h+1) r}-2\right)}$ we get $|V(\Gamma(R))|=\frac{2}{p^{(h+1) r}-2}|E|$.

Acknowledgements. Supported by University of Kabianga Research Fund.

\section{References}

[1] Bidwel J. Automorphisms of direct product of finite groups, 86, 481-489 Arch. Math, (2007).

[2] Chikunji J., Automorphisms of completely primary finite rings of characteristic p, Colloq. Math. 111, 91-113 (2008).

[3] Lao H. Mude, Owino M. Oduor, Ojiema M. Onyango Automorphisms of zero divisor graphs of Galois rings. 8, 401- 406, (2019).

[4] Ojiema M. Onyango, Owino M. Oduor, Odhiambo P. Olieche, Automorphisms of unit groups of power four radical zero completely primary finite rings,1, 11-25,(2016).

[5] Owino M. Oduor, Ojiema M. Onyango., Mmasi Eliud., Units of commutative completely primary finite rings of characteristic $p^{n}$, International Journal of Algebra, vol.7, No. 6, (2016).

[6] Raghavendran R, Finite associative rings Compositio Math 21, 195-229, (1969). 